\title{
Energy Dependent Inverse Scattering
}

\author{
By \\ Yutaka KAMIMURA \\ (Tokyo University of Marine Science and Technology, Japan) \\ Dedicated to Professor Kazuo Okamoto on his 60th birthday
}

\begin{abstract}
The paper discusses the inverse scattering problem to recover the potentials of an energy dependent Schrödinger equation from the scattering data. A necessary and sufficient condition for a given function to be the scattering data is obtained. The potential can be recovered by a simple formula from the solution of a Marchenko type equation with a function determined from the scattering data.

Key Words and Phrases. Energy dependent Schrödinger equation, Inverse scattering problem, Marchenko equation.

2000 Mathematics Subject Classification Numbers. 81U40, 34A55, 45E10.
\end{abstract}

\section{Introduction}

As a generalization of the time-independent Klein-Gordon equation, Cornille [5] considered an energy dependent Schrödinger equation

$$
f^{\prime \prime}+\left[k^{2}-(U(x)+2 E Q(x))\right] f=0,
$$

where $k$ is the wave number, $E=\sqrt{k^{2}+m^{2}}$ is the energy, $Q$ and $U$ are static potentials for a particle (anti-particle) of mass $m$. He derived a Marchenko equation for the Schrödinger equation and suggested the validity of the Marchenko method (refer to Marchenko [14], see also Chadan and Sabatier [4]) for inverse scattering problems in relativistic quantum mechanics.

For $m=0$ corresponding to a particle of zero mass such as the photon, the radial s-wave Schrödinger equation becomes

$$
f^{\prime \prime}+\left[k^{2}-(U(x)+2 k Q(x))\right] f=0, \quad 0 \leq x<\infty,
$$

and, as a special case $U(x)=-Q(x)^{2}$, it gives

$$
f^{\prime \prime}+(k-Q)^{2} f=0,
$$

which is the time-independent Klein-Gordon equation; it can be deduced from the quantization $p=-i \hbar(d / d x)$ ( $\hbar$ denotes the Plank constant) for the momentum $p$ in the relativistic energy equality 


$$
(E-Q)^{2}=(p c)^{2}+\left(m c^{2}\right)^{2}
$$

with $m=0$, after a scale-transformation (adopting the natural units).

Jaulent and Jean [9] and Jaulent [6,7] treated the inverse scattering problem for the equation (1.1) and have established a procedure by which the potentials $U$ and $Q$ are recovered from the scattering data, under the assumption that $U, Q$ are real-valued, differentiable functions belonging to $L^{1}(0, \infty)$ together with the derivatives and that there are no bound states. They derived a nonlinear differential equation for $\int_{x}^{\infty} Q(\eta) d \eta$ from the Marchenko equation and, based upon it, established a sufficient condition for a given function to be the scattering data, in other words, proved a pair of potentials $(Q, U)$ is uniquely determined from the scattering data if it satisfies the condition.

The purpose of the present paper is to make clear the inverse scattering transform (correspondence from scattering data to a pair of potentials) by employing values on the diagonal line (a function on the half-line) of the solution to a new Marchenko-type equation in place of the nonlinear differential equation. Our method enables us to establish a necessary and sufficient condition (in terms of the function on the half-line) for a given function to be the scattering data, and moreover, yields an explicit expression of the inverse scattering transform (see (1.5) and (1.6) in Theorem 1.1) that gives a direct method for calculating potentials from the given function.

The inverse scattering problem we treat in this paper is to recover a pair $(Q, U)$ of potentials from the coefficient $S(k)$ appearing in the scattering solution

$$
\psi(x, k)=e^{-i k x}-S(k) e^{i k x}+o(1), \quad x \rightarrow \infty,
$$

of (1.1) that vanishes at $x=0$. The function $S(k)$ on the real axis is referred to as the scattering data for a pair $(Q, U)$. Under the assumptions that $Q(x)$, $U(x)$ are real-valued and that

$$
Q(x),(1+x) U(x) \in L^{1}(0, \infty),
$$

the scattering solution $\psi(x, k)$ is expressed as

$$
\psi(x, k)=\overline{f(x, k)}-S(k) f(x, k)
$$

in terms of the Jost solution $f(x, k)$, namely the (unique) solution of (1.1) with the asymptotics

$$
f(x, k)=e^{i k x}[1+o(1)], \quad x \rightarrow \infty,
$$

defined for each $k$ in the upper half-plane $\operatorname{Im} k \geq 0$. Hence the scattering data $S(k)$ is written as 


$$
S(k)=\frac{\overline{f(0, k)}}{f(0, k)} .
$$

Therefore our problem consists in exploring the inverse of the scattering transform

$$
(Q, U) \mapsto S
$$

Since the Wronskian $W[f(x, k), \overline{f(x, k)}]$ of solutions $f(x, k)$ and $\overline{f(x, k)}$ is computed as

$$
W[f(x, k), \overline{f(x, k)}]=-2 i k, \quad k \in \boldsymbol{R},
$$

it follows that $f(0, k) \neq 0$ for $k \in \boldsymbol{R} \backslash\{0\}$. Clearly we have

$$
|S(k)|=1 \text {. }
$$

We assume that $f(0,0) \neq 0$. Then, by the expression

$$
f(x, k)=e^{i \int_{x}^{\infty} Q(\eta) d \eta} e^{i k x}+\int_{x}^{\infty} A(x, t) e^{i k t} d t, \quad \operatorname{Im} k \geq 0,
$$

where $A(x, \cdot) \in L^{1}(x, \infty)$ (see [11, Lemma 2.1]) and the Wiener-Lévy theorem, it follows that $S(k)$ is expressed as

$$
S(k)=C+\int_{-\infty}^{\infty} F(t) e^{-i k t} d t
$$

in terms of a complex constant $C$ of absolute value 1 and a function $F(t) \in L^{1}(\boldsymbol{R})$.

We shall treat the case where there are neither quasi-bound states nor bound states. Namely we assume that

$$
f(0,0) \neq 0 ; \quad f(0, k) \neq 0, \quad \operatorname{Im} k>0 .
$$

In view of the argument principle, this is equivalent to

$$
\text { ind } f(0, k):=\frac{1}{2 \pi}[\arg f(0, k)]_{-\infty}^{\infty}=0 .
$$

Hence, from the definition (1.2), we have:

$$
\text { ind } S(k)=0 \text {. }
$$

Following the previous paper [11], we employ a Marchenko-type equation:

$$
\overline{\Delta(x, t)}+\int_{x}^{\infty} \Delta(x, r) F(r+t) d r+\int_{x}^{\infty} F(r+t) d r=0, \quad x \leq t .
$$


A standard analysis (refer to [11, Lemma 4.2]) shows that, under the conditions $(\mathrm{S} 1)-(\mathrm{S} 3)$, this equation has a unique solution $\Delta(x, \cdot)$ in the space $B C[x, \infty)$ for each $x \geq 0$. Here $B C[x, \infty)$ denotes the space of bounded, continuous functions on the interval $[x, \infty)$. Moreover it can be proved (see Lemma 2.1 in Section 2) that $1+\Delta(x, x) \neq 0$ for each $x \geq 0$. We can deduce (see Section 4) the following relation between the constant $C$ in the expression (1.3) and the values on the diagonal line of the solution $\Delta(x, t)$ :

$$
e^{2 i[\arg (1+\Delta(x, x))]_{0}^{\infty}}=C .
$$

Notice that $C, F(t)$ and $\Delta(x, t)$ are determined uniquely from $S(k)$, and hence, (S4) is a condition on the function $S(k)$.

Though we shall later establish a result (see Theorem 4.1) that needs no differentiability condition on $Q$, we assume at this stage that $Q(x)$ is differentiable and that

$$
(1+x) Q^{\prime}(x) \in L^{1}(0, \infty) .
$$

Then it can be shown (see Jaulent and Jean [9, Theorem 5.1]) that the part of the function $F(t)$ in $0 \leq t<\infty$ is differentiable and satisfies

$$
(1+t) F^{\prime}(t) \in L^{1}(0, \infty) .
$$

The following theorem that will be established in the subsequent sections asserts that the collection of the conditions (S1)-(S5) is not merely necessary but also sufficient for a function $S(k)$ to be the scattering data for a pair $(Q, U)$ satisfying the conditions (A1)-(A3):

Theorem 1.1. A function $S(k)$ on $\boldsymbol{R}$ is the scattering data for a pair $(Q, U)$ of real-valued functions $Q(x), U(x)$ satisfying (A1)-(A3) if and only if $S(k)$ satisfies $(\mathrm{S} 1)-(\mathrm{S} 5)$. Then the functions $Q(x), U(x)$ are determined by

$$
\begin{aligned}
& Q(x)=-\frac{d}{d x}(\operatorname{Im} \log (1+\Delta(x, x))), \\
& U(x)=\left(\frac{d}{d x}(\operatorname{Re} \log (1+\Delta(x, x)))\right)^{2}-\frac{d^{2}}{d x^{2}}(\operatorname{Re} \log (1+\Delta(x, x))),
\end{aligned}
$$

where $\Delta(x, t)$ is a solution of (1.4).

This theorem shows that the scattering transform $(Q, U) \mapsto S$ gives a one to one correspondence from the set of pairs $(Q, U)$ of functions satisfying (A1)(A3) to the set of functions $S$ satisfying (S1)-(S5) and gives the inverse of it in an explicit and simple form. It is of worth to mention here that the expression (1.3) can be rewritten by an integration by parts as 


$$
f(x, k)=f(x, 0) e^{i k x}-i k \int_{x}^{\infty} K(x, t) e^{i k t} d t, \quad \operatorname{Im} k \geq 0,
$$

where $K(x, t):=-\int_{t}^{\infty} A(x, \eta) d \eta$ and, our fundamental function $\Delta(x, t)$ is connected with $A(x, t)$ through $K(x, t)$ by

$$
K(x, t)=\frac{\Delta(x, t)}{|1+\Delta(x, x)|},
$$

which will be mentioned in Remark 3.4. Equation (1.4) is derived by rewriting the Marchenko equation for $A(x, t)$ (see Jaulent [6, equation (1.23)]) along this connection.

Since $Q(x) \equiv 0$ if and only if the solution $\Delta(x, t)$ of (1.4) is real-valued (equivalent to $\overline{S(k)}=S(-k)$, also to that $F(t)$ is real-valued), the condition (S4) is satisfied automatically with $C=1$ in the case of $Q(x) \equiv 0$. In this case, (1.6) coincides with the well-known recovery formula

$$
U(x)=-2 \frac{d}{d x} A(x, x)
$$

(see Marchenko [14, Chap. 3]) for the classical inverse scattering theory in nonrelativistic quantum mechanics.

We illustrate our results via an example:

$$
S(k):=\frac{(k+\alpha)(k+\bar{\beta})}{(k+\bar{\alpha})(k+\beta)}, \quad k \in \boldsymbol{R},
$$

where $\alpha, \beta$ are complex numbers with $\operatorname{Im} \alpha, \operatorname{Im} \beta>0$. An elementary observation shows that this function $S(k)$ satisfies the conditions (S1)-(S3) and (S5). Moreover, for this $S(k)$, the solution of the equation (1.4) is given in concrete by means of elementary functions and, as will be shown in Section 5, a function $S(k)$ in (1.8) satisfies the condition (S4), namely, it is the scattering data for a pair $(Q, U)$ of real-valued functions $Q, U$ satisfying (A1)-(A3), if and only if $\beta=c \alpha$ with $c>0$. In particular, if (and only if) $\alpha, \beta$ are purely imaginary, $Q(x)$ becomes 0 identically.

Besides in relativistic quantum mechanics, the inverse scattering theory for the energy dependent Schrödinger equation is applied in many areas of physics. Sattinger and Szmigielski [16] simplified the theory developed by Jaulent and Jean [10] in one dimensional, energy dependent Schrödinger equation to solve a nonlinear evolution equation. Jaulent [8] applied the theory to a class of inverse problems in absorbing media with waves propagating. This problem is reduced to the inverse scattering for energy dependent Schrödinger equation in the case where $U(x)$ is real and $Q(x)$ is purely imaginary. Aktosun, Klaus and van der Mee [2, 3] developed the inverse scattering theory in this case. Moreover, 
motivated by a problem on a long-wave water equation, Kaup [13] discussed the inverse scattering problem of the equation

$$
f^{\prime \prime}+\left[E^{2}-(U(x)+2 k Q(x))\right] f=0, \quad-\infty<x<\infty
$$

with $E=\sqrt{k^{2}+m^{2}}$, real $U(x)$ and purely imaginary $Q(x)$. Sattinger and Szmigielski [17] studied the problem as a Riemann-Hilbert problem; and van der Mee and Pivovarchik [18] studied it by the Marchenko integral equation approach. Furthermore the author [12] applied the inverse scattering theory for (1.1) with $U=0$ and $Q(x)$ real to an inverse problem of determining the advection term of an advection-diffusion equation.

\section{Fundamental function}

Under the assumption (A1), the Jost solution $f(x, k)$ of $(1.1)$ is holomorphic with respect to $k$ in the upper half-plane $\operatorname{Im} k>0$ and is continuous in its closure $\operatorname{Im} k \geq 0$. The solution is represented as (1.7) through a function $K(x, t) \in B C[x, \infty)$. It can be shown (see [11, Lemma 4.1]) that if the conditions (A1) and (A2) are fulfilled then $K(x, t)$ satisfies

$$
\overline{K(x, t)}+\int_{x}^{\infty} K(x, r) F(r+t) d r+f(x, 0) \int_{x}^{\infty} F(r+t) d r=0, \quad x \leq t,
$$

where $F$ is a function in $L^{1}(\boldsymbol{R})$ used in the expression (S2). Replacing $f(x, 0)$ in (2.1) by 1 , we deduce integral equation (1.4). If $S(k)$ defined by (S2) with a function $F(t) \in L^{1}(\boldsymbol{R})$ and a complex number $C$ of absolute value 1 satisfies (S1) and (S3), then the equation (1.4), which is viewed as an equation for unknown function of $t$ in the interval $[x, \infty)$ for each fixed $x \geq 0$, admits a unique solution $\Delta(x, \cdot)$ in the space $B C[x, \infty)$ (refer to [11, Lemma 4.2]). The solution $\Delta(x, t)$ plays a fundamental role for our discussion. In this section we shall study some properties of the function $\Delta(x, t)$.

Lemma 2.1. Let $F \in L^{1}(\boldsymbol{R})$ and suppose that, for $F$, there is a complex number $C$ with $|C|=1$ such that a function $S(k)$ defined by $(S 2)$ satisfies (S1) and (S3). Then:

(i) There exists a constant $M$ independent of $x$, $t$ such that

$$
|\Delta(x, t)| \leq M \int_{x+t}^{\infty}|F(r)| d r, \quad x \leq t .
$$

In particular,

$$
\lim _{t \rightarrow \infty} \Delta(x, t)=0
$$


(ii) For each $x \geq 0, \Delta(x, t)$ has the partial derivative $\Delta_{t}(x, t)$ with respect to $t$. The derivative $\Delta_{t}(x, \cdot)$ belongs to $L^{1}(x, \infty)$ for each $x \geq 0$ and satisfies

$$
\overline{\Delta_{t}(x, t)}-\int_{x}^{\infty} \Delta_{t}(x, r) F(r+t) d r=(1+\Delta(x, x)) F(x+t), \quad x \leq t .
$$

(iii) For each $x \geq 0,1+\Delta(x, x) \neq 0$.

Proof. Assertion (i) can be proved by the same argument as in Agranovich and Marchenko [1, Lemma 4.1.1]. For the proof of (ii), let $F_{n}(t) \quad(n=1,2, \ldots)$ be a sequence of functions in $L^{1}(\boldsymbol{R})$ with $F_{n}^{\prime}(t) \in L^{1}(\boldsymbol{R})$ such that $F_{n} \rightarrow F$ in the norm of $L^{1}(\boldsymbol{R})$. Then, for sufficiently large $n$, each of the equations

$$
\overline{\Delta^{n}(x, t)}+\int_{x}^{\infty} \Delta^{n}(x, r) F_{n}(r+t) d r+\int_{x}^{\infty} F_{n}(r+t) d r=0, \quad x \leq t
$$

has a unique solution $\Delta^{n}(x, t)$ in the space $B C[x, \infty)$, and, in view of $(2.2)$,

$$
\lim _{n \rightarrow \infty} \Delta^{n}(x, t)=\Delta(x, t)
$$

in the norm of $B C[x, \infty)$. Because of $F_{n}^{\prime}(t) \in L^{1}(\boldsymbol{R}), \Delta^{n}(x, t)$ is differentiable in $t$ and its derivative $\Delta_{t}^{n}(x, t)$ belongs to $B C[x, \infty)$. Moreover, as is verified by an integration by parts, $\Delta_{t}^{n}(x, t)$ satisfies

$$
\overline{\Delta_{t}^{n}(x, t)}-\int_{x}^{\infty} \Delta_{t}^{n}(x, r) F_{n}(r+t) d r=\left(1+\Delta^{n}(x, x)\right) F_{n}(x+t), \quad x \leq t .
$$

On the other hand, the equation

$$
\overline{B(x, t)}-\int_{x}^{\infty} B(x, r) F(r+t) d r=(1+\Delta(x, x)) F(x+t), \quad x \leq t
$$

admits a unique solution $B(x, t)$ in $L^{1}(x, \infty)$. Hence, for sufficiently large $n$, each of the equations

$$
B^{n}(x, t)-\int_{x}^{\infty} \overline{B^{n}(x, r)} F_{n}(r+t) d r=(1+\Delta(x, x)) F_{n}(x+t), \quad x \leq t
$$

also admits a unique solution $B^{n}(x, t)$ in $L^{1}(x, \infty)$, and

$$
\lim _{n \rightarrow \infty} B_{t}^{n}(x, t)=B(x, t)
$$

in the norm of $L^{1}(x, \infty)$. Since the assumption $F_{n}^{\prime}(t) \in L^{1}(\boldsymbol{R})$ implies $F_{n}(t) \in B C(\boldsymbol{R})$, it follows that the solution $B^{n}(x, t)$ belongs to $B C[x, \infty)$ as well as to $L^{1}(x, \infty)$, and therefore, that $B^{n}(x, t)=\Delta_{t}^{n}(x, t)$. This shows that, for $x \leq t$, 


$$
\begin{aligned}
\Delta(x, t)-\Delta(x, x) & =\lim _{n \rightarrow \infty}\left(\Delta^{n}(x, t)-\Delta^{n}(x, x)\right) \\
& =\lim _{n \rightarrow \infty} \int_{x}^{t} B^{n}(x, s) d s=\int_{x}^{t} B(x, s) d s .
\end{aligned}
$$

Hence the function $\Delta(x, t)$ is differentiable in $t$ and its derivative satisfies (2.4).

We shall prove the assertion (iii) by contradiction. If $1+\Delta(x, x)=0$ for some $x \geq 0$ then, by $(2.4)$, the derivative $\Delta_{t}(x, \cdot) \in L^{1}(x, \infty)$ satisfies

$$
\overline{\Delta_{t}(x, t)}-\int_{x}^{\infty} \Delta_{t}(x, r) F(r+t) d r=0, \quad x \leq t .
$$

Since this equation has no non-trivial solutions in $L^{1}(x, \infty)$ (see [11, Lemma B.1]), we find that $\Delta_{t}(x, \cdot)=0$ in $L^{1}(x, \infty)$. This, together with (2.3), shows that $\Delta(x, x)=0$, which is incompatible with $1+\Delta(x, x)=0$. Therefore $1+\Delta(x, x) \neq 0$ for any $x \geq 0$.

In order to examine the function $\Delta(x, t)$ in more detail, we suppose that $F(t)$ is bounded and continuous on the positive half interval.

Lemma 2.2. Suppose that, in addition to the assumption in Lemma 2.1, the part of $F(t)$ in $0 \leq t<\infty$ belongs to $B C[0, \infty)$. Then:

(i) The solution $\Delta(x, t)$ of (2.1) has the partial derivative $\Delta_{x}(x, t)$ as well as $\Delta_{t}(x, t)$. The derivatives $\Delta_{x}(x, \cdot), \Delta_{t}(x, \cdot)$ belong to $L^{1}(x, \infty) \cap B C[x, \infty)$ for each $x \geq 0$.

(ii) The function

$$
\frac{\Delta_{x}(x, x)-\Delta_{t}(x, x)}{1+\Delta(x, x)}, \quad x \geq 0,
$$

is real-valued.

(iii) For each $x, y \geq 0$, the function above is differentiable and its derivative satisfies

$$
\frac{d}{d x}\left(\frac{\Delta_{x}(x, x+y)-\Delta_{t}(x, x+y)}{1+\Delta(x, x)}\right)=\overline{\left(\frac{\frac{d}{d x} \Delta(x, x)}{1+\Delta(x, x)}\right)}\left(\frac{\frac{d}{d x} \Delta(x, x+y)}{1+\Delta(x, x)}\right) .
$$

(iv) For each $x \geq 0$,

$$
\frac{d}{d x}\left(\frac{\Delta_{x}(x, x)-\Delta_{t}(x, x)}{1+\Delta(x, x)}\right)=\left|\frac{\frac{d}{d x} \Delta(x, x)}{1+\Delta(x, x)}\right|^{2}
$$


Proof. By a similar argument to in the proof of Lemma 2.1, we can show that the function $\Delta(x, t)$ has the partial derivative $\Delta_{x}(x, t)$ with respect to $x$, which belongs to $L^{1}(x, \infty)$ for each $x \geq 0$ and satisfies

$$
\overline{\Delta_{x}(x, t)}+\int_{x}^{\infty} \Delta_{x}(x, r) F(r+t) d r=(1+\Delta(x, x)) F(x+t), \quad x \leq t .
$$

From this equation and the assumption $F(t) \in B C[0, \infty)$, it follows that $\Delta_{x}(x, \cdot) \in B C[x, \infty)$ as well as $\Delta_{x}(x, \cdot) \in L^{1}(0, \infty)$. Similarly, with the aid of (2.4), we can show that $\Delta_{t}(x, \cdot) \in B C[x, \infty)$.

In order to prove the assertion (ii), we set

$$
w(x)=\frac{1+\Delta(x, x)}{\overline{1+\Delta(x, x)}}, \quad x \geq 0,
$$

and define an operator $\mathbf{F}_{x}$ by

$$
\left(\mathbf{F}_{x} \varphi\right)(y)=w(x) \int_{0}^{\infty} F(s+y+2 x) \varphi(s) d s .
$$

It is known (see Marchenko [14, Lemma 3.3.1]) that $\mathbf{F}_{x}$ is a compact operator on $L^{2}(0, \infty)$.

In view of $(2.5)$, the function

$$
p(x, y):=\frac{\Delta_{x}(x, x+y)}{1+\Delta(x, x)}, \quad x, y \geq 0
$$

satisfies

$$
\overline{p(x, y)}+w(x) \int_{0}^{\infty} p(x, s) F(s+y+2 x) d s=w(x) F(y+2 x), \quad y \geq 0 .
$$

Since $p(x, \cdot) \in L^{1}(0, \infty)$ and $F(\cdot+2 x) \in L^{1}(0, \infty) \cap B C[0, \infty)$, the function $p(x, \cdot)$ also belongs to $B C[0, \infty)$. Therefore, by observing $L^{1}(0, \infty) \cap B C[0, \infty) \subset$ $L^{2}(0, \infty)$, it follows that $p(x, \cdot) \in L^{2}(0, \infty)$. Hence the equation above is written as an equation in $L^{2}(0, \infty)$ :

$$
\left(J+\mathbf{F}_{x}\right) p(x, y)=w(x) F(y+2 x),
$$

where $J$ is the conjugation operator, that is, an operator defined by $J \varphi=\bar{\varphi}$.

By a standard Fourier analysis (see e.g. [11, Appendix B]), one can show that if $\tau \geq 1$ then the operator $\tau J-\mathbf{F}_{x}$ on $L^{2}(0, \infty)$ is injective. Accordingly, in view of the identity

$$
\lambda I-\mathbf{F}_{x}^{*} \mathbf{F}_{x}=J i\left(\sqrt{\lambda} J-\mathbf{F}_{x}\right) i J\left(\sqrt{\lambda} J-\mathbf{F}_{x}\right), \quad \lambda \geq 0,
$$


if $\lambda \geq 1$ then $\lambda I-\mathbf{F}^{*} \mathbf{F}$ is also injective. This shows that each eigenvalue $\lambda$ of the positive, compact, selfadjoint operator $\mathbf{F}_{x}^{*} \mathbf{F}_{x}$ on $L^{2}(0, \infty)$ is smaller than 1 , and hence that

$$
\left\|\mathbf{F}_{x}\right\|=\sup _{\|\varphi\|=1}\left(\mathbf{F}_{x} \varphi, \mathbf{F}_{x} \varphi\right)=\sup _{\|\varphi\|=1}\left(\mathbf{F}_{x}^{*} \mathbf{F}_{x} \varphi, \varphi\right)<1 .
$$

Therefore the Neumann series $\sum_{n=0}^{\infty}\left( \pm J \mathbf{F}_{x}\right)^{n}$ converges and gives the inverse of $\left(I \mp J \mathbf{F}_{x}\right)$. In particular we obtain

$$
\begin{aligned}
& \frac{\Delta_{x}(x, x+y)}{1+\Delta(x, x)}=\left(I+J \mathbf{F}_{x}\right)^{-1} J w(x) F(y+2 x), \\
& \frac{\Delta_{t}(x, x+y)}{1+\Delta(x, x)}=\left(I-J \mathbf{F}_{x}\right)^{-1} J w(x) F(y+2 x),
\end{aligned}
$$

which yields

$$
\frac{\Delta_{x}(x, x+y)-\Delta_{t}(x, x+y)}{1+\Delta(x, x)}=-2\left(I-\mathbf{F}_{x}^{*} \mathbf{F}_{x}\right)^{-1} \mathbf{F}_{x}^{*} w(x) F(y+2 x) .
$$

We now set $\mathbf{G}_{x}:=\mathbf{F}_{x}^{*} \mathbf{F}_{x}$. Then the operator $\mathbf{G}_{x}$ on $L^{2}(0, \infty)$ is written as

$$
\mathbf{G}_{x} \varphi(y)=\int_{0}^{\infty} G(y, z ; x) \varphi(z) d z
$$

where $G(y, z ; x)$ is a function on $[0, \infty) \times[0, \infty)$ defined by

$$
G(y, z ; x)=\int_{0}^{\infty} \overline{F(s+y+2 x)} F(s+z+2 x) d s \quad\left(=\int_{2 x}^{\infty} \overline{F(s+y)} F(s+z) d s\right) .
$$

Moreover we obtain

$$
\frac{\Delta_{x}(x, x+y)-\Delta_{t}(x, x+y)}{1+\Delta(x, x)}=-2\left(I-\mathbf{G}_{x}\right)^{-1} G(y, 0 ; x) .
$$

Since $\|\mathbf{G}\|<1$, the inverse $\left(I-\mathbf{G}_{x}\right)^{-1}$ is given by the Neumann series

$$
\left(I-\mathbf{G}_{x}\right)^{-1}=I+\sum_{n=0}^{\infty} \mathbf{G}_{x}^{n}
$$

Let $G_{n}(y, z ; x), n=1,2, \ldots$, be a sequence of functions defined by

$$
G_{n}(y, z ; x)=\mathbf{G}_{x}^{n-1} G(y, z ; x) .
$$

Then $\mathbf{G}_{x}^{n}$ is integral operators expressed as

$$
\mathbf{G}_{x}^{n} \varphi(y)=\int_{0}^{\infty} G_{n}(y, z ; x) \varphi(z) d z
$$


and $G_{n}(y, z ; x)$ satisfies, for $n=1,2, \ldots$,

$$
G_{n+1}(y, z ; x)=\int_{0}^{\infty} G(y, s ; x) G_{n}(s, z ; x) d s=\int_{0}^{\infty} G_{n}(y, s ; x) G(s, z ; x) d s .
$$

Hence, by induction, we get the symmetry property:

$$
G_{n}(y, z ; x)=\overline{G_{n}(z, y ; x)}, \quad n=1,2, \ldots
$$

By assumption, the part of $F(t)$ in $0 \leq t<\infty$ belongs to $L^{2}(0, \infty)$. Hence, by Young's inequality, we can verify that

$$
\|G(\cdot, z ; x)\|^{2}=\int_{0}^{\infty}|G(y, z ; x)|^{2} d y \leq\left(\int_{2 x}^{\infty}|F(s)| d s\right)^{2}\left(\int_{z+2 x}^{\infty}|F(s)|^{2} d s\right) .
$$

Therefore, noting $G(y, z ; x)=\overline{G(z, y ; x)}$, we have

$$
\begin{aligned}
\left|\sum_{n=\ell}^{m} G_{n}(y, z ; x)\right| & =\left|\int_{0}^{\infty} G(y, s ; x) \sum_{n=\ell-2}^{m-2} \mathbf{G}_{x}^{n} G(s, z ; x) d s\right| \\
& \leq\|G(\cdot, y ; x)\|\left\|\sum_{n=\ell-2}^{m-2} \mathbf{G}_{x}^{n} G(\cdot, z ; x)\right\| \\
& \leq\|G(\cdot, y ; x)\|\left\|\sum_{n=\ell-2}^{m-2} \mathbf{G}_{x}^{n}\right\|\|G(\cdot, z ; x)\| \rightarrow 0
\end{aligned}
$$

as $m>\ell \rightarrow \infty$, uniformly in $y, z$. This shows that a function $H(y, z ; x)$ defined by

$$
H(y, z ; x):=\sum_{n=1}^{\infty} G_{n}(y, z ; x)
$$

becomes a continuous function on $y, z \geq 0$. The symmetry property

$$
H(y, z ; x)=\overline{H(z, y ; x)}
$$

of $H(y, z ; x)$ is passed on from that of $G_{n}(y, z ; x)$.

By the definition of $G_{n}(y, z, x)$ we have

$$
H(\cdot, z ; x)=\sum_{n=0}^{\infty} \mathbf{G}_{x}^{n} G(\cdot, z ; x) .
$$

Therefore $H(y, z ; x)$ satisfies

$$
\left(I-\mathbf{G}_{x}\right) H(\cdot, z ; x)=G(\cdot, z ; x)
$$


for each $z \geq 0$. Moreover the inverse operator $\left(I-\mathbf{G}_{x}\right)^{-1}$ of $\left(I-\mathbf{G}_{x}\right)$ is an integral operator given by

$$
\left(I-\mathbf{G}_{x}\right)^{-1} \varphi(y)=\varphi(y)+\int_{0}^{\infty} H(y, s ; x) \varphi(s) d s .
$$

Formula (2.8), together with (2.11), shows that

$$
\frac{\Delta_{x}(x, x+y)-\Delta_{t}(x, x+y)}{1+\Delta(x, x)}=-2 H(y, 0 ; x) .
$$

In particular, we obtain

$$
\frac{\Delta_{x}(x, x)-\Delta_{t}(x, x)}{1+\Delta(x, x)}=-2 H(0,0 ; x) .
$$

This, together with (2.10), proves the assertion (ii).

Since the function $G(y, z ; x)$ defined by $(2.7)$ is differentiable with respect to $x$, it follows from $(2.11)$ that $H(y, 0 ; x)$ is also differentiable with respect to $x$ and the derivative $H_{x}(y, 0 ; x)$ is given by

$$
H_{x}(y, 0, x)=\left(I-\mathbf{G}_{x}\right)^{-1}\left\{G_{x}(y, 0 ; x)+\int_{0}^{\infty} G_{x}(y, s ; x) H(s, 0 ; x) d s\right\} .
$$

Rewriting the right-hand side by means of (2.7), (2.12) and (2.10), we have

$$
\begin{aligned}
-\frac{1}{2} H_{x}(y, 0 ; x)= & \left(I-\mathbf{G}_{x}\right)^{-1}\{\overline{F(y+2 x)} F(2 x) \\
& \left.+\overline{F(y+2 x)} \int_{0}^{\infty} F(s+2 x) H(s, 0 ; x) d s\right\} \\
= & \overline{F(y+2 x)} F(2 x)+\int_{0}^{\infty} H(y, s ; x) \overline{F(s+2 x)} d s F(2 x) \\
& +\overline{F(y+2 x)} \int_{0}^{\infty} F(s+2 x) H(s, 0 ; x) d s \\
& +\int_{0}^{\infty} H(y, s ; x) \overline{F(s+2 x)} d s \int_{0}^{\infty} F(s+2 x) H(s, 0 ; x) d s \\
= & \left(F(2 x)+\int_{0}^{\infty} H(s, 0 ; x) F(s+2 x) d s\right) \\
& \times \overline{\left(F(y+2 x)+\int_{0}^{\infty} H(s, y ; x) F(s+2 x) d s\right)}
\end{aligned}
$$


which, combined with (2.13), leads to

$$
\begin{aligned}
\frac{d}{d x}( & \left.\frac{\Delta_{x}(x, x+y)-\Delta_{t}(x, x+y)}{1+\Delta(x, x)}\right) \\
= & 4\left(F(2 x)+\int_{0}^{\infty} H(s, 0 ; x) F(s+2 x) d s\right) \\
& \times \overline{\left(F(y+2 x)+\int_{0}^{\infty} H(s, y ; x) F(s+2 x) d s\right) .}
\end{aligned}
$$

Finally we compute the right-hand side of the equality in the assertion (iii). By (2.6) and (2.12) we obtain

$$
\begin{aligned}
\frac{\frac{d}{d x} \Delta(x, x+y)}{1+\Delta(x, x)} & =\frac{\Delta_{x}(x, x+y)+\Delta_{t}(x, x+y)}{1+\Delta(x, x)} \\
& =\left\{\left(I+J \mathbf{F}_{x}\right)^{-1}+\left(I-J \mathbf{F}_{x}\right)^{-1}\right\} J w(x) F(y+2 x) \\
& =2\left(I+J \mathbf{F}_{x}\right)^{-1}\left(I-J \mathbf{F}_{x}\right)^{-1} J w(x) F(y+2 x) \\
& =2\left(I-\mathbf{G}_{x}\right)^{-1} J w(x) F(y+2 x) \\
& =2 \overline{w(x)} \overline{\left(F(y+2 x)+\int_{0}^{\infty} H(s, y ; x) F(s+2 x) d s\right)} .
\end{aligned}
$$

Inserting this to the right-hand side in (2.14) and noting $|w(x)|=1$ prove the assertion (iii). Assertion (iv) is direct from (iii) by setting $y=0$.

As a consequence of the above proof, we have

Corollary 2.3. Under the assumption as in Lemma 2.2, the following formula holds:

$$
\overline{\left(\frac{\frac{d}{d x} \Delta(x, x)}{1+\Delta(x, x)}\right)}=2 w(x)\left(F(2 x)+\int_{0}^{\infty} H(y, 0 ; x) F(y+2 x) d y\right),
$$

where $|w(x)|=1$ and $H(y, z ; x)$ is estimated as

$$
|H(y, z ; x)| \leq M\left(\int_{2 x}^{\infty}|F(s)|^{2} d s\right)
$$

with a constant $M$ independent of $y, z$. 
Proof. On setting $y=0$ in (2.15), we obtain (2.16). By means of (2.9) with $\ell=2, m=\infty$, we obtain

$$
\begin{aligned}
\left|\sum_{n=2}^{\infty} G_{n}(y, z ; x)\right| & \leq M_{0}\|G(\cdot, y ; x)\|\|G(\cdot, z ; x)\| \\
& \leq\left(\int_{2 x}^{\infty}|F(s)| d s\right)^{2}\left(\int_{y+2 x}^{\infty}|F(s)|^{2} d s\right)^{1 / 2}\left(\int_{z+2 x}^{\infty}|F(s)|^{2} d s\right)^{1 / 2} \\
& \leq M_{1}\left(\int_{y+2 x}^{\infty}|F(s)|^{2} d s\right)^{1 / 2}\left(\int_{z+2 x}^{\infty}|F(s)|^{2} d s\right)^{1 / 2}
\end{aligned}
$$

where $M_{1}=M_{0}\|F\|^{2}$. Moreover, by (2.7), we get

$$
|G(y, z ; x)| \leq\left(\int_{y+2 x}^{\infty}|F(s)|^{2} d s\right)^{1 / 2}\left(\int_{z+2 x}^{\infty}|F(s)|^{2} d s\right)^{1 / 2} .
$$

Hence, by taking $M=M_{1}+1$, we arrive at (2.17).

\section{Construction of potentials}

In this section we shall construct a pair $(Q, U)$ of potentials in the Schrödinger equation (1.1) from the fundamental function $\Delta(x, t)$ explored in the previous section. Throughout this section, let $F(t)$ be a function in $L^{1}(\boldsymbol{R})$ whose part in $[0, \infty)$ belongs to $B C[0, \infty)$, let $S(k)$ be a function satisfying the conditions $(\mathrm{S} 1)-(\mathrm{S} 3)$, and let $\Delta(x, t)$ be the solution of (1.4).

Our discussion is based upon the recovering formula

$$
\frac{2 \Delta_{t}(x, x)}{1+\Delta(x, x)}=\int_{x}^{\infty}\left[U(r)+Q(r)^{2}\right] d r-i Q(x), \quad 0 \leq x<\infty
$$

which was developed in the previous paper [11, equation (1.11)]). Following this formula we define functions $Q(x), U(x)$ by

$$
\begin{aligned}
& Q(x)=-2 \operatorname{Im} \frac{\Delta_{t}(x, x)}{1+\Delta(x, x)}, \\
& U(x)=-2 \frac{d}{d x}\left(\operatorname{Re} \frac{\Delta_{t}(x, x)}{1+\Delta(x, x)}\right)-Q(x)^{2} .
\end{aligned}
$$

Clearly $Q(x), U(x)$ are real-valued functions. Our next step requires that we rewrite these definitions:

Lemma 3.1. The functions $Q(x), U(x)$ defined by (3.2), (3.3) are rewritten as 


$$
\begin{aligned}
& Q(x)=-\operatorname{Im} \frac{\frac{d}{d x} \Delta(x, x)}{1+\Delta(x, x)}, \\
& U(x)=\left(\operatorname{Re} \frac{\frac{d}{d x} \Delta(x, x)}{1+\Delta(x, x)}\right)^{2}-\frac{d}{d x}\left(\operatorname{Re} \frac{\frac{d}{d x} \Delta(x, x)}{1+\Delta(x, x)}\right) .
\end{aligned}
$$

Proof. Assertion (ii) of Lemma 2.2 enables us to compute

$$
\begin{aligned}
Q(x) & =-2 \operatorname{Im} \frac{\Delta_{t}(x, x)}{1+\Delta(x, x)}-\operatorname{Im} \frac{\Delta_{x}(x, x)-\Delta_{t}(x, x)}{1+\Delta(x, x)} \\
& =-\operatorname{Im} \frac{\Delta_{x}(x, x)+\Delta_{t}(x, x)}{1+\Delta(x, x)} .
\end{aligned}
$$

This yields (3.4).

By the definition (3.3) and (3.4), we have

$$
\begin{aligned}
U(x) & =-2 \frac{d}{d x}\left(\operatorname{Re} \frac{\Delta_{t}(x, x)}{1+\Delta(x, x)}\right)-\left(\operatorname{Im} \frac{\frac{d}{d x} \Delta(x, x)}{1+\Delta(x, x)}\right)^{2} \\
& =-2 \frac{d}{d x}\left(\operatorname{Re} \frac{\Delta_{t}(x, x)}{1+\Delta(x, x)}\right)-\left|\frac{\frac{d}{d x} \Delta(x, x)}{1+\Delta(x, x)}\right|^{2}+\left(\operatorname{Re} \frac{\frac{d}{d x} \Delta(x, x)}{1+\Delta(x, x)}\right)^{2} .
\end{aligned}
$$

But, use of the assertions (ii) and (iv) of Lemma 2.2 shows that

$$
\begin{aligned}
& -2 \frac{d}{d x}\left(\operatorname{Re} \frac{\Delta_{t}(x, x)}{1+\Delta(x, x)}\right)-\left|\frac{\frac{d}{d x} \Delta(x, x)}{1+\Delta(x, x)}\right|^{2} \\
& \quad=-2 \frac{d}{d x}\left(\operatorname{Re} \frac{\Delta_{t}(x, x)}{1+\Delta(x, x)}\right)-\frac{d}{d x}\left(\operatorname{Re} \frac{\Delta_{x}(x, x)-\Delta_{t}(x, x)}{1+\Delta(x, x)}\right) \\
& =-\frac{d}{d x}\left(\operatorname{Re} \frac{\frac{d}{d x} \Delta(x, x)}{1+\Delta(x, x)}\right) .
\end{aligned}
$$

Therefore we arrive at (3.5).

We now define a function $f_{0}(x)$ by 


$$
f_{0}(x)=\frac{1}{|1+\Delta(x, x)|} .
$$

It is evident from (2.2) that $f_{0}(x) \rightarrow 1$ as $x \rightarrow \infty$. Moreover (3.6) is rewritten as

$$
f_{0}(x)=\frac{1}{1+\Delta(x, x)} e^{i \int_{x}^{\infty} Q(\eta) d \eta}
$$

because

$$
Q(x)=-\operatorname{Im} \frac{d}{d x} \log (1+\Delta(x, x))=-\frac{d}{d x} \arg (1+\Delta(x, x)) .
$$

Lemma 3.2. Let $Q(x), U(x)$ be functions defined by (3.4), (3.5) and let $f_{0}(x)$ be a function defined by (3.6). Then:

(i) The function $f_{0}(x)$ satisfies $f_{0}^{\prime \prime}(x)=U(x) f_{0}(x)$.

(ii) For $x, y \geq 0$,

$$
\frac{d}{d x}\left(\Delta_{x}(x, x+y)-\Delta_{t}(x, x+y)\right)=2 i Q(x) \Delta_{t}(x, x+y)-2 \frac{f_{0}^{\prime}(x)}{f_{0}(x)} \Delta_{x}(x, x+y) .
$$

Proof. Differentiating (3.7) we have

$$
f_{0}^{\prime}(x)=\left(-\frac{\frac{d}{d x} \Delta(x, x)}{1+\Delta(x, x)}-i Q(x)\right) f_{0}(x)
$$

which, combined with (3.4), yields

$$
f_{0}^{\prime}(x)=-\left(\operatorname{Re} \frac{\frac{d}{d x} \Delta(x, x)}{1+\Delta(x, x)}\right) f_{0}(x) .
$$

Differentiating this again we have

$$
f_{0}^{\prime \prime}(x)=\left\{\left(\operatorname{Re} \frac{\frac{d}{d x} \Delta(x, x)}{1+\Delta(x, x)}\right)^{2}-\frac{d}{d x}\left(\operatorname{Re} \frac{\frac{d}{d x} \Delta(x, x)}{1+\Delta(x, x)}\right)\right\} f_{0}(x),
$$

and using (3.5) we complete the proof of the assertion (i).

By the assertion (iii) of Lemma 2.2 and (3.4), we have

$$
\begin{aligned}
& \frac{d}{d x}\left(\Delta_{x}(x, x+y)-\Delta_{t}(x, x+y)\right) \\
& \quad=\frac{d}{d x}\left(\frac{\Delta_{x}(x, x+y)-\Delta_{t}(x, x+y)}{1+\Delta(x, x)}(1+\Delta(x, x))\right)
\end{aligned}
$$




$$
\begin{aligned}
= & \left(\operatorname{Re} \frac{\frac{d}{d x} \Delta(x, x)}{1+\Delta(x, x)}-i \operatorname{Im} \frac{\frac{d}{d x} \Delta(x, x)}{1+\Delta(x, x)}\right) \frac{d}{d x} \Delta(x, x+y) \\
& +\frac{\Delta_{x}(x, x+y)-\Delta_{t}(x, x+y)}{1+\Delta(x, x)} \frac{d}{d x} \Delta(x, x) \\
= & \left.\operatorname{Re} \frac{\frac{d}{d x} \Delta(x, x)}{1+\Delta(x, x)}\right) \frac{d}{d x} \Delta(x, x+y)+i Q(x) \frac{d}{d x} \Delta(x, x+y) \\
& +\left(\frac{\frac{d}{d x} \Delta(x, x)}{1+\Delta(x, x)}\right)\left(\Delta_{x}(x, x+y)-\Delta_{t}(x, x+y)\right) \\
= & 2 i Q(x) \Delta_{t}(x, x+y)+2\left(\operatorname{Re} \frac{\frac{d}{d x} \Delta(x, x)}{1+\Delta(x, x)}\right) \Delta_{x}(x, x+y) .
\end{aligned}
$$

This, together with (3.10), proves the assertion (ii).

We next define a function $f(x, k)$ for each $k$ in the upper half-plane $\operatorname{Im} k \geq 0$ by

$$
f(x, k)=f_{0}(x)\left(e^{i k x}-i k \int_{x}^{\infty} \Delta(x, t) e^{i k t} d t\right) .
$$

The following asserts that this function $f(x, k)$ is nothing but the Jost solution of differential equation (1.1) with potentials $(Q(x), U(x))$ defined by (3.4), (3.5).

Lemma 3.3. For each $k$ in $\operatorname{Im} k \geq 0$, the function $f(x, k)$ defined by (3.11) is a solution of $(1.1)$ satisfying $f(x, k)=e^{i k x}[1+o(1)]$ as $x \rightarrow \infty$.

Proof. By (i) of Lemma 3.2, we have

$$
\begin{aligned}
f^{\prime \prime}(x, k)-U(x) f(x, k)= & 2 i k f_{0}^{\prime}(x) e^{i k x}-2 i k f_{0}^{\prime}(x)\left(\int_{x}^{\infty} \Delta(x, t) e^{i k t} d t\right)^{\prime} \\
& -k^{2} f_{0}(x) e^{i k x}-i k f_{0}(x)\left(\int_{x}^{\infty} \Delta(x, t) e^{i k t} d t\right)^{\prime \prime}
\end{aligned}
$$

A calculation shows that

$$
\left(\int_{x}^{\infty} \Delta(x, t) e^{i k t} d t\right)^{\prime}=-\Delta(x, x) e^{i k x}+\int_{x}^{\infty} \Delta_{x}(x, t) e^{i k t} d t .
$$


Since the right-hand side of this equality can be rewritten as

$$
-2 \Delta(x, x) e^{i k x}+\int_{0}^{\infty}\left(\Delta_{x}(x, x+y)-\Delta_{t}(x, x+y)\right) e^{i k y} d y e^{i k x}-i k \int_{x}^{\infty} \Delta(x, t) e^{i k t} d t,
$$

it follows from (ii) of Lemma 3.2 that

$$
\begin{aligned}
\left(\int_{x}^{\infty} \Delta(x, t) e^{i k t} d t\right)^{\prime \prime}= & -i k \Delta(x, x) e^{i k x}-2\left(\frac{d}{d x} \Delta(x, x)\right) e^{i k x} \\
& +\int_{0}^{\infty}\left(2 i Q(x) \Delta_{t}(x, x+y)-2 \frac{f_{0}^{\prime}(x)}{f_{0}(x)} \Delta_{x}(x, x+y)\right) e^{i k y} d y e^{i k x} \\
& -i k \int_{x}^{\infty} \Delta_{t}(x, t) e^{i k t} d t .
\end{aligned}
$$

Hence, performing an integration by parts to the last term, we obtain

$$
\begin{aligned}
\left(\int_{x}^{\infty} \Delta(x, t) e^{i k t} d t\right)^{\prime \prime}= & -2\left(\frac{d}{d x} \Delta(x, x)\right) e^{i k x}-k^{2} \int_{x}^{\infty} \Delta(x, t) e^{i k t} d t \\
& +2 i Q(x) \int_{x}^{\infty} \Delta_{t}(x, t) e^{i k t} d t \\
& -2 \frac{f_{0}^{\prime}(x)}{f_{0}(x)} \int_{x}^{\infty} \Delta_{x}(x, t) e^{i k t} d t
\end{aligned}
$$

Insertion of (3.13), (3.14) in (3.12) leads to

$$
\begin{aligned}
f^{\prime \prime}(x, k)-U(x) f(x, k)= & 2 i k\left\{f_{0}^{\prime}(x)(1+\Delta(x, x))+f_{0}(x)\left(\frac{d}{d x} \Delta(x, x)\right)\right\} e^{i k x} \\
& -k^{2} f_{0}(x)\left(e^{i k x}-i k \int_{x}^{\infty} \Delta(x, t) e^{i k t} d t\right) \\
& +2 k Q(x) f_{0}(x) \int_{x}^{\infty} \Delta_{t}(x, t) e^{i k t} d t .
\end{aligned}
$$

Performing an integration by parts to the last term and noting definition (3.11), we can rewrite this as

$$
\begin{aligned}
f^{\prime \prime}(x, k)-U(x) f(x, k)= & 2 i k\left\{f_{0}^{\prime}(x)(1+\Delta(x, x))+f_{0}(x)\left(\frac{d}{d x} \Delta(x, x)\right)\right\} e^{i k x} \\
& -k^{2} f(x, k)-2 k Q(x) f_{0}(x)(1+\Delta(x, x)) e^{i k x} \\
& +2 k Q(x) f(x, k) .
\end{aligned}
$$

But, by (3.9), 


$$
f_{0}^{\prime}(x)(1+\Delta(x, x))+f_{0}(x)\left(\frac{d}{d x} \Delta(x, x)\right)=-i Q(x) f_{0}(x)(1+\Delta(x, x)) .
$$

Hence we arrive at

$$
f^{\prime \prime}(x, k)-U(x) f(x, k)=-k^{2} f(x, k)+2 k Q(x) f(x, k) .
$$

The proof is complete.

Remark 3.4. If we set

$$
K(x, t)=f_{0}(x) \Delta(x, t)=\frac{\Delta(x, t)}{|1+\Delta(x, x)|},
$$

then the equation (3.11) is rewritten as (1.7). In particular, if $Q(x) \equiv 0$ then $\Delta(x, t)$ is real-valued (refer to [11, Theorem 4.3]) and hence

$$
K(x, t)=\frac{\Delta(x, t)}{1+\Delta(x, x)} .
$$

Accordingly if $Q(x) \equiv 0$ then, by (3.3),

$$
U(x)=-2 \frac{d}{d x} K_{t}(x, x) .
$$

\section{Scattering transform}

In this section we shall establish theorems which show that the scattering transform $(Q, U) \mapsto S$ becomes a one-to-one correspondence in an appropriate function spaces framework of potentials and scattering data. One of such theorems is Theorem 1.1 given in Section 1. We here present the proof.

Proof of Theorem 1.1. We shall first show that, given $Q(x), U(x)$ satisfying (A1)-(A3), the function $S(k)$ defined by (1.2) satisfies (S1)-(S5). Clearly, $S(k)$ satisfies (S1). By the expression (1.3) we have

$$
f(0, k)=e^{i \int_{0}^{\infty} Q(\eta) d \eta}+\int_{0}^{\infty} A(0, t) e^{i k t} d t
$$

where $A(0, t) \in L^{1}(0, \infty)$. This expression and the assumption that $f(0,0) \neq 0$ in (A2) allow us to apply the Wiener-Lévy theorem (see e.g., Paley and Wiener $[15$, p. 63]). Then, after a convolution calculation, it follows that $S(k)$ is represented as

$$
S(k)=e^{-2 i \int_{0}^{\infty} Q(\eta) d \eta}+\int_{-\infty}^{\infty} F(t) e^{-i k t} d t, \quad k \in \boldsymbol{R}
$$

in terms of a function $F(t)$ in $L^{1}(\boldsymbol{R})$. Thus $S(k)$ satisfies (S2). 
By the argument principle, the index ind $f(0, k)$ gives the number of zeros, counted with multiplicities, of $f(0, k)$ in the region $\operatorname{Im} k>0$, since $f(0, k)$ is holomorphic in the region and is continuous in its closure. Hence, by the assumption (A2), the index

$$
\text { ind } f(0, k):=\frac{1}{2 \pi}[\arg f(0, k)]_{-\infty}^{\infty}
$$

equals zero. This, combined with the definition (1.2), yields (S3).

By the Riemann-Lebesgue lemma, the constant $C$ in (S2) is uniquely determined from $S(k)$ as a limit of it when $|k| \rightarrow \infty$. Accordingly, the constant $C$ in (S2) can be connected with $Q$ by

$$
C=e^{-2 i \int_{0}^{\infty} Q(\eta) d \eta}
$$

In view of the uniqueness theorem for the Fourier transform, the function $F(t)$ in (S2) is also uniquely determined from $S(k)$, and so, the fundamental function $\Delta(x, t)$ is uniquely determined as the solution of (1.4). It is evident that if $Q(x)$ satisfies the condition (A3) then $Q(x) \in B C[0, \infty)$, and therefore, as was shown in [11, Theorem 1.1], $Q(x)$ is recovered by (3.1). Consequently, by Lemma 3.1, $Q(x)$ is expressed as (3.4), in other words, as

$$
Q(x)=-\frac{d}{d x} \arg (1+\Delta(x, x)) .
$$

This, together with (4.2), leads to (S4). Property (S5) follows from Jaulent and Jean [9, Theorem 5.1].

We shall next show that if a function $S(k)$ on $\boldsymbol{R}$ satisfies $(\mathrm{S} 1)-(\mathrm{S} 5)$ then $S(k)$ is the scattering data for a pair $(Q, U)$ of real-valued functions satisfying (A1)-(A3). First we note that, by Lemma 2.1, the solution $\Delta(x, t)$ of $(1.4)$ satisfies $1+\Delta(x, x) \neq 0$ for $x \geq 0$, which enables us to define $Q(x), U(x)$ by (3.2), (3.3) or equivalently, by (3.4), (3.5). Since $F(t), F^{\prime}(t) \in L^{1}(0, \infty)$ we have

$$
F(t)=-\int_{t}^{\infty} F^{\prime}(s) d s
$$

Hence, if we set

$$
F^{\#}(t)=\int_{t}^{\infty}\left|F^{\prime}(s)\right| d s,
$$

then

$$
|F(t)| \leq F^{\#}(t), \quad F^{\#}(t) \text { is a nonincreasing function in } L^{1}(0, \infty),
$$

because 


$$
\int_{0}^{\infty} F^{\#}(t) d t=\int_{0}^{\infty} d t \int_{t}^{\infty}\left|F^{\prime}(s)\right| d s=\int_{0}^{\infty} s\left|F^{\prime}(s)\right| d s<\infty .
$$

By means of the function $F^{\#}(t)$, it follows from (2.17) that the function $H(y, z ; x)$ in $(2.16)$ is estimated as

$$
|H(y, z ; x)| \leq M \int_{2 x}^{\infty}|F(s)|^{2} d s \leq M F^{\#}(2 x) \int_{2 x}^{\infty} F^{\#}(s) d s \leq M_{1} F^{\#}(2 x) .
$$

Moreover, by (2.16), we have

$$
\left|\frac{\frac{d}{d x} \Delta(x, x)}{1+\Delta(x, x)}\right|=2\left|F(2 x)+\int_{0}^{\infty} H(y, 0 ; x) F(y+2 x) d y\right| \leq M_{2} F^{\#}(2 x),
$$

with some constant $M_{2}$. This, combined with (4.3), shows that

$$
p(x):=\frac{\frac{d}{d x} \Delta(x, x)}{1+\Delta(x, x)}
$$

belongs to $L^{1}(0, \infty)$. Hence, by (3.4), $Q(x) \in L^{1}(0, \infty)$.

In view of (2.13), (2.14), (4.4), there exists a constant $M$ such that

$$
\left|H_{x}(y, 0 ; x)\right| \leq M\left(F^{\#}(2 x)\right)^{2} .
$$

This, together with (4.4), yields

$$
\begin{aligned}
& \left|\frac{d}{d x} \int_{0}^{\infty} H(0, s ; x) F(s+2 x) d s\right| \\
& \quad \leq \int_{0}^{\infty}\left|H_{x}(0, s ; x)\right||F(s+2 x)| d r+\int_{0}^{\infty}|H(0, s ; x)|\left|F^{\prime}(s+2 x)\right| d r \\
& \quad \leq M \int_{0}^{\infty} F^{\#}(2 x)^{2}|F(s+2 x)| d s+M_{1} F^{\#}(2 x) \int_{2 x}^{\infty}\left|F^{\prime}(\eta)\right| d \eta \\
& \quad \leq M_{3} F^{\#}(2 x)^{2}
\end{aligned}
$$

where $x F^{\#}(2 x)^{2} \in L^{1}(0, \infty)$ since

$$
\int_{0}^{\infty} 2 x F^{\#}(2 x)^{2} d x=\int_{0}^{\infty} d x \int_{2 x}^{\infty} F^{\#}(\eta)^{2} d \eta \leq \int_{0}^{\infty} F^{\#}(2 x) d x \int_{2 x}^{\infty} F^{\#}(\eta) d \eta<\infty .
$$

It is easy to see from this observation and (2.16) that the function $p(x)$ defined by (4.6) is differentiable and its derivative $p^{\prime}(x)$ satisfies $(1+x) p^{\prime}(x) \in L^{1}(0, \infty)$. In particular, $Q(x)$ satisfies (A3). 
By $(1+x) p^{\prime}(x) \in L^{1}(0, \infty)$ we have

$$
\begin{aligned}
|p(x)| \leq \int_{x}^{\infty}\left|p^{\prime}(\eta)\right| d \eta=: p^{\#}(x) & \\
\int_{0}^{\infty} x|p(x)|^{2} d x & =\int_{0}^{\infty} d x \int_{x}^{\infty}|p(\eta)|^{2} \eta \leq \int_{0}^{\infty} d x \int_{x}^{\infty} p^{\#}(\eta)^{2} d \eta \\
\leq & \int_{0}^{\infty} p^{\#}(x) d x \int_{x}^{\infty} p^{\#}(\eta) d \eta<\infty .
\end{aligned}
$$

Therefore the function $U(x)=(\operatorname{Re} p(x))^{2}-\operatorname{Re} p^{\prime}(x)$ satisfies $(1+x) U(x) \in$ $L^{1}(0, \infty)$. In this way, we have proved that the functions $Q(x), U(x)$ defined by (3.4), (3.5) satisfies (A1) and (A3).

We now define a function $f_{0}(x)$ by (3.6) and, with the aid of $f_{0}(x)$, define $f(x, k)$ for $x \geq 0, \operatorname{Im} k \geq 0$ by (3.11). Then, by Lemma 3.3, $f(x, k)$ is the Jost solution of (1.1). Notice that $f_{0}(x) \neq 0$ for $x \geq 0$ and hence, by (3.11), $f(0,0) \neq 0$. To show that the scattering data for this pair $(Q, U)$ coincides with $S(k)$, namely, to prove (1.2), we define a function $A(x, t)$ for $0 \leq x \leq t<\infty$ by

$$
A(x, t)=f_{0}(x) \Delta_{t}(x, t)\left(=\frac{\Delta_{t}(x, t)}{|1+\Delta(x, x)|}\right) .
$$

Then, by performing an integration by parts to (3.11) and taking (3.7) into account, it follows that the Jost solution $f(x, k)$ is expressed as

$$
f(x, k)=e^{i \int_{x}^{\infty} Q(\eta) d \eta} e^{i k x}+\int_{x}^{\infty} A(x, t) e^{i k t} d t .
$$

Moreover, by (3.7) and (2.4), $A(x, t)$ satisfies

$$
\overline{A(x, t)}-\int_{x}^{\infty} A(x, r) F(r+t) d r-e^{i \int_{x}^{\infty} Q(\eta) d \eta} F(x+t)=0, \quad x \leq t .
$$

Because of (3.8), the assumption (S4) is written as (4.2). Hence we obtain

$$
\begin{aligned}
\overline{f(0, k)} & -S(k) f(0, k) \\
= & \left(e^{-i \int_{0}^{\infty} Q(\eta) d \eta}+\int_{0}^{\infty} \overline{A(0, t)} e^{-i k t} d t\right) \\
& -\left(e^{-2 i \int_{0}^{\infty} Q(\eta) d \eta}+\int_{-\infty}^{\infty} F(t) e^{-i k t} d t\right)\left(e^{i \int_{0}^{\infty} Q(\eta) d \eta}+\int_{-\infty}^{0} A(0,-t) e^{-i k t} d t\right) .
\end{aligned}
$$

By the convolution theorem this can be written as

$$
\overline{f(0, k)}-S(k) f(0, k)=\int_{-\infty}^{\infty} \Phi(t) e^{-i k t} d t
$$


where $\Phi(t)$ is a function on $\boldsymbol{R}$ defined by

$$
\begin{aligned}
\Phi(t)= & \overline{A(0, t)}-e^{-2 i \int_{0}^{\infty} Q(\eta) d \eta} A(0,-t) \\
& -\int_{0}^{\infty} A(0, r) F(r+t) d r-e^{i \int_{0}^{\infty} Q(\eta) d \eta} F(t) .
\end{aligned}
$$

It should be mentioned that $A(0, t)=0$ for $t<0$ and $A(0,-t)=0$ for $t>0$. In what follows we shall prove that $\Phi(t)=0$ for almost every $t \in \boldsymbol{R}$. In the case of $t>0$, the assertion $\Phi(t)=0$ is equivalent to (4.10) with $x=0$. Thus we obtain $\Phi(t)=0$ for $t>0$.

In order to show that $\Phi(t)=0$ for $t<0$, we multiply both sides of (4.12) by $\overline{F(x+t)}$ and integrate on $\boldsymbol{R}$ with respect to $t$. Then, since $\Phi(t)=0$ for $t>0$ and $A(0, t)=0$ for $t<0$, we get

$$
\begin{aligned}
& \int_{-\infty}^{0} \Phi(t) \overline{F(x+t)} d t=\int_{0}^{\infty} \overline{A(0, t)} \overline{F(x+t)} d t \\
& -e^{-2 i \int_{0}^{\infty} Q(\eta) d \eta} \int_{-\infty}^{0} A(0,-t) \overline{F(x+t)} d t \\
& -\int_{-\infty}^{\infty} \overline{F(x+t)} d t \int_{0}^{\infty} A(0, r) F(r+t) d r
\end{aligned}
$$

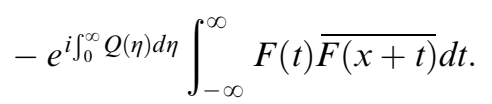

We rewrite the third term of the right-hand side. By noting that $(\mathrm{S} 1)$, that is, $S(k) \overline{S(k)}=1$ and $(\mathrm{S} 2)$ yield

$$
\int_{-\infty}^{\infty} F(t) \overline{F(x+t)} d t=-\bar{C} F(-x)-C \overline{F(x)}, \quad-\infty<x<\infty,
$$

it follows from (4.2) that

$$
\begin{aligned}
& -\int_{-\infty}^{\infty} \overline{F(x+t)} d t \int_{0}^{\infty} A(0, r) F(r+t) d r=-\int_{0}^{\infty} A(0, r) d r \int_{-\infty}^{\infty} F(t) \overline{F(x-r+t)} d t \\
& =\int_{0}^{\infty} A(0, r)\{\bar{C} F(r-x)+C \overline{F(x-r)}\} d r \\
& =\bar{C} \int_{0}^{\infty} A(0, r) F(r-x) d r
\end{aligned}
$$

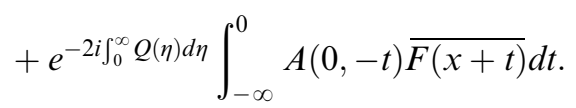

Hence we have 


$$
\begin{aligned}
\int_{-\infty}^{0} \Phi(t) \overline{F(x+t)} d t= & \int_{0}^{\infty} \overline{A(0, t)} \overline{F(x+t)} d t+e^{i \int_{0}^{\infty} Q(\eta) d \eta} \bar{C} F(-x) \\
& +\bar{C} \int_{0}^{\infty} A(0, r) F(r-x) d r+e^{i \int_{0}^{\infty} Q(\eta) d \eta} C \overline{F(x)}
\end{aligned}
$$

But, by the definition (4.12) and (4.2), we find that the right-hand side of this equality equals

$$
-\overline{\Phi(x)}-\bar{C} \Phi(-x)
$$

Therefore we have

$$
\overline{\Phi(x)}+\bar{C} \Phi(-x)+\int_{-\infty}^{0} \Phi(t) \overline{F(x+t)} d t=0, \quad-\infty<x<\infty .
$$

Since, as has been shown already, $\Phi(x)=0$ for $x>0$, this yields

$$
\Phi(x)+\int_{-\infty}^{0} \overline{\Phi(t)} F(x+t) d t=0, \quad-\infty<x<0 .
$$

This shows that $\Phi(x)=0$ because a solution of it is unique (see [11, Lemma B.1]). Hence, in view of (4.11), we obtain (1.2). By (1.2) and the assumption $(\mathrm{S} 3)$, it follows that ind $f(0, k)=0$. This, together with the argument principle, shows that $f(0, k) \neq 0$ for $k$ in $\operatorname{Im} k>0$.

At the present time we worked with the differentiability of the potential $Q(x)$ and the function $F(t)$ in the expression (S2). To broaden the framework beyond the extent of differentiable functions, we introduce the following condition on $Q(x)$ :

$\left(\mathrm{A} 3^{\#}\right) \quad Q(x)$ is a continuous function on $[0, \infty)$ and satisfies

$$
|Q(x)| \leq Q^{\#}(x), \quad 0 \leq x<\infty,
$$

with some nonincreasing function $Q^{\#}(x) \in L^{1}(0, \infty)$.

It is evident that if $Q(x)$ satisfies the condition (A3\#) then $Q(x) \in$ $B C[0, \infty)$. Moreover, as is seen in analogy to (4.3), if $Q(x) \in L^{1}(0, \infty)$ satisfies the condition (A3) then it satisfies $\left(\mathrm{A}^{*}{ }^{\#}\right)$.

Corresponding to the condition $\left(\mathrm{A} 3^{\#}\right)$ we replace the condition (S5) by the following condition on $F(t)$ :

$\left(\mathrm{S}^{\#}\right)$ The part of $F(t)$ in $0 \leq t<\infty$ is a continuous function on $[0, \infty)$ and satisfies

$$
|F(t)| \leq F^{\#}(t), \quad 0 \leq t<\infty,
$$


with some nonincreasing function $F^{\#}(t) \in L^{1}(0, \infty)$; and moreover the function

$$
\operatorname{Re} \frac{\frac{d}{d x} \Delta(x, x)}{1+\Delta(x, x)}
$$

defined by means of the solution $\Delta(x, y)$ of (1.4) is differentiable and its derivative satisfies

$$
(1+x) \frac{d}{d x}\left(\operatorname{Re} \frac{\frac{d}{d x} \Delta(x, x)}{1+\Delta(x, x)}\right) \in L^{1}(0, \infty) .
$$

The same conclusion as in Theorem 1.1 can be reached using $\left(\mathrm{A}^{\#}{ }^{\#}\right)$ and $\left(\mathrm{S}^{\#}\right)$ in place of $(\mathrm{A} 3)$ and $(\mathrm{S} 5)$ respectively:

Theorem 4.1. A function $S(k)$ on $\boldsymbol{R}$ is the scattering data for a pair $(Q, U)$ of real-valued functions $Q, U$ satisfying (A1), (A2), (A3\#) if and only if $S(k)$ satisfies (S1)-(S4), (S5\#).

Proof. We shall first show that, given $Q(x), U(x)$ satisfying (A1), (A2), $\left(\mathrm{A} 3^{\#}\right)$, the function $S(k)$ defined by $(1.2)$ satisfies $(\mathrm{S} 1)-(\mathrm{S} 4),\left(\mathrm{S} 5^{\#}\right)$. Since the discussion concerning $(\mathrm{S} 1)-(\mathrm{S} 4)$ in the proof of Theorem 1.1 applies verbatim, we shall only prove $\left(\mathrm{S}^{\#}{ }^{\#}\right)$. Let

$$
\sigma^{\#}(x)=\int_{x}^{\infty}|U(\eta)| d \eta+Q^{\#}(x) .
$$

Then, by a similar argument to that in [11, proof of Lemma 2.1], it follows that the function $A(x, t)$ in (1.3) becomes a continuous function in $0 \leq x \leq t<\infty$ and is estimated as

$$
|A(x, t)| \leq M \sigma^{\#}\left(\frac{x+t}{2}\right),
$$

where $M$ is a constant independent of $x, t$. Moreover $A(x, t)$ satisfies (4.10), which yields

$$
e^{i \int_{x}^{\infty} Q(\eta) d \eta} F(2 x)=\overline{A(x, x)}-\int_{x}^{\infty} A(x, 2 \eta-x) F(2 \eta) d r, \quad 0 \leq x<\infty .
$$

It is clear from this equality that $F(t)$ is continuous for $t \geq 0$. Moreover, by (4.15), we have, for $x \geq 0$, 


$$
\begin{aligned}
|F(2 x)| & \leq M \sigma^{\#}(x)+\int_{x}^{\infty} M \sigma^{\#}(\eta)|F(2 \eta)| d \eta \\
& \leq M \sigma^{\#}(x)+M \sigma^{\#}(x) \int_{x}^{\infty}|F(2 \eta)| d \eta \\
& \leq M^{\prime} \sigma^{\#}(x) .
\end{aligned}
$$

Hence, by setting $F^{\#}(t)=M^{\prime} \sigma^{\#}(t / 2)$ and noting $\sigma^{\#} \in L^{1}(0, \infty)$, we conclude that the part of $F(t)$ in $0 \leq t<\infty$ satisfies (4.13).

Since $Q(x) \in B C[0, \infty)$, the potentials $Q(x)$ and $U(x)$ must satisfy the recovering formula (3.1). Hence, using (3.2) and Lemma 2.2, in particular the assertions (ii), (iv) there, we obtain

$$
\begin{aligned}
\int_{x}^{\infty}\left[U(\eta)+Q(\eta)^{2}\right] d \eta & =2 \frac{\Delta_{t}(x, x)}{1+\Delta(x, x)}-2 i \operatorname{Im} \frac{\Delta_{t}(x, x)}{1+\Delta(x, x)} \\
& =2 \operatorname{Re} \frac{\Delta_{t}(x, x)}{1+\Delta(x, x)} \\
& =-\operatorname{Re} \frac{\Delta_{x}(x, x)-\Delta_{t}(x, x)}{1+\Delta(x, x)}+\operatorname{Re} \frac{\frac{d}{d x} \Delta(x, x)}{1+\Delta(x, x)} \\
& =\int_{x}^{\infty}\left|\frac{\frac{d}{d r} \Delta(r, r)}{1+\Delta(r, r)}\right|^{2} d r+\operatorname{Re} \frac{\frac{d}{d x} \Delta(x, x)}{1+\Delta(x, x)} .
\end{aligned}
$$

Therefore the function

$$
\operatorname{Re} \frac{\frac{d}{d x} \Delta(x, x)}{1+\Delta(x, x)}
$$

is differentiable and, in view of (3.4), its derivative is given by

$$
\frac{d}{d x}\left(\operatorname{Re} \frac{\frac{d}{d x} \Delta(x, x)}{1+\Delta(x, x)}\right)=-U(x)+\left(\operatorname{Re} \frac{\frac{d}{d x} \Delta(x, x)}{1+\Delta(x, x)}\right)^{2}
$$

By (4.5) we have

$$
\left(\operatorname{Re} \frac{\frac{d}{d x} \Delta(x, x)}{1+\Delta(x, x)}\right)^{2} \leq\left|\frac{\frac{d}{d x} \Delta(x, x)}{1+\Delta(x, x)}\right|^{2} \leq M_{2}^{2} F^{\#}(2 x)^{2} .
$$


This, together with the estimate (4.9), shows that

$$
(1+x)\left(\operatorname{Re} \frac{\frac{d}{d x} \Delta(x, x)}{1+\Delta(x, x)}\right)^{2} \in L^{1}(0, \infty) .
$$

Accordingly, by (4.16) and the assumption (A1), we get (4.14). In this way we have proved $\left(\mathrm{S} 5^{\#}\right)$.

We shall next show that if a function $S(k)$ on $\boldsymbol{R}$ satisfies $(\mathrm{S} 1)-(\mathrm{S} 4),\left(\mathrm{S} 5^{\#}\right)$ then $S(k)$ is the scattering data for a pair $(Q, U)$ of real-valued functions satisfying (A1), (A2), (A3*). Let $Q(x)$ be a function defined by (3.4). Then, by (4.5), we have

$$
|Q(x)| \leq\left|\frac{\frac{d}{d x} \Delta(x, x)}{1+\Delta(x, x)}\right| \leq M_{2} F^{\#}(2 x) .
$$

This leads to $\left(\mathrm{A} 3^{\#}\right)$. With the aid of the assumption $\left(\mathrm{S} 5^{\#}\right)$ we can define $U(x)$ by (3.5). Then, by (4.17) and the assumption (4.14), we find that $(1+x) U(x) \in L^{1}(0, \infty)$. By the exactly same argument as in the proof of Theorem 1.1 we show that the function $f(x, k)$ defined by (3.11) satisfies (1.2) and (A2). The proof is complete.

\section{Example}

This section is devoted to an investigation of an example. Let $\alpha, \beta$ be complex numbers whose imaginary parts are positive, and let

$$
S(k):=\frac{(k+\alpha)(k+\bar{\beta})}{(k+\bar{\alpha})(k+\beta)}, \quad k \in \boldsymbol{R} .
$$

Then, clearly, $|S(k)|=1$ for any $k \in \boldsymbol{R}$. Moreover

$$
\text { ind } S(k)=\text { ind } \frac{k+\alpha}{k+\bar{\alpha}}-\text { ind } \frac{k+\beta}{k+\bar{\beta}}=1-1=0 \text {. }
$$

Furthermore, since

$$
S(k)=1+A \int_{0}^{\infty} e^{-i \bar{\alpha} t} e^{-i k t} d t+B \int_{-\infty}^{0} e^{-i \beta t} e^{-i k t} d t
$$

with

$$
A=2(\operatorname{Im} \alpha) \frac{\bar{\beta}-\bar{\alpha}}{\bar{\alpha}-\beta}, \quad B=2(\operatorname{Im} \beta) \frac{\beta-\alpha}{\bar{\alpha}-\beta},
$$


the function $S(k)$ is written as

$$
S(k)=1+\int_{-\infty}^{\infty} F(t) e^{-i k t} d t
$$

where

$$
F(t)= \begin{cases}A e^{-i \bar{\alpha} \cdot t} & \text { for } t>0 \\ B e^{-i \beta t} & \text { for } t<0\end{cases}
$$

Thus the function $S(k)$ defined in (5.1) satisfies the conditions (S1)-(S3) and (S5) provided that $\operatorname{Im} \alpha, \operatorname{Im} \beta>0$.

The Marchenko-type equation (1.4) for the function in (5.1) becomes

$$
\overline{\Delta(x, t)}+A \int_{x}^{\infty} \Delta(x, r) e^{-i \bar{\alpha} r} d r e^{-i \bar{\alpha} t}+\frac{A}{i \bar{\alpha}} e^{-i \bar{\alpha}(x+t)}=0, \quad x \leq t .
$$

To solve this equation, we set

$$
g(x):=\int_{x}^{\infty} \Delta(x, r) e^{-i \bar{\alpha} r} d r .
$$

Then we get

$$
\overline{\Delta(x, t)}=-A\left(g(x)+\frac{1}{i \bar{\alpha}} e^{-i \bar{\alpha} x}\right) e^{-i \bar{\alpha} t}
$$

Insertion of the complex conjugate of the right-hand side to (5.6) yields

$$
g(x)=-\frac{\bar{A}}{2(\operatorname{Im} \alpha)} e^{-2(\operatorname{Im} \alpha) x}\left(\overline{g(x)}+\frac{i}{\alpha} e^{i \alpha x}\right) .
$$

Taking the complex conjugate of this equation and substituting it to the righthand of the equation, we obtain

$$
(1-e(x)) g(x)=e(x) \frac{1}{i \bar{\alpha}} e^{-i \bar{\alpha} x}+\frac{\bar{A}}{2(\operatorname{Im} \alpha)} e^{-2(\operatorname{Im} \alpha) x} \frac{1}{i \alpha} e^{i \alpha x},
$$

where we set

$$
e(x):=\frac{|A|^{2}}{4(\operatorname{Im} \alpha)^{2}} e^{-4(\operatorname{Im} \alpha) x} .
$$

Since $\operatorname{Im} \alpha, \operatorname{Im} \beta>0$, it follows from (5.2) that

$$
e(x)=\left|\frac{\bar{\beta}-\bar{\alpha}}{\bar{\alpha}-\beta}\right|^{2} e^{-4(\operatorname{Im} \alpha) x} \leq\left|\frac{\bar{\beta}-\bar{\alpha}}{\bar{\alpha}-\beta}\right|^{2}<1 .
$$


Hence the function $g(x)$ can be determined by (5.8), and a computation shows that

$$
g(x)+\frac{1}{i \bar{\alpha}} e^{-i \bar{\alpha} x}=\frac{1}{1-e(x)}\left(\frac{\bar{A}}{2(\operatorname{Im} \alpha)} e^{-2(\operatorname{Im} \alpha) x} \frac{1}{i \alpha} e^{i \alpha x}+\frac{1}{i \bar{\alpha}} e^{-i \bar{\alpha} x}\right) .
$$

By substituting this in (5.7), the equation (5.5) is solved as

$$
\Delta(x, t)=\frac{1}{1-e(x)}\left(\frac{|A|^{2}}{2(\operatorname{Im} \alpha)} e^{-2(\operatorname{Im} \alpha) x} \frac{1}{i \bar{\alpha}} e^{-i \bar{\alpha} x}+\frac{\bar{A}}{i \alpha} e^{i \alpha x}\right) e^{i \alpha t} .
$$

From this we can draw the following conclusion:

Proposition 5.1. Let $\operatorname{Im} \alpha, \operatorname{Im} \beta>0$. Then a function $S(k)$ in (5.1) is the scattering data for a pair $(U, Q)$ of real-valued functions satisfying (A1)-(A3) if and only if $\beta=c \alpha$ with $c>0$.

Proof. We have already shown that $S(k)$ satisfies the conditions (S1)-(S3) and (S5). Hence, by Theorem 1.1, it suffices to show that $S(k)$ satisfies (S4) if and only if $\beta=c \alpha$ with $c>0$. By (5.9) we have

$$
1+\Delta(x, x)=\frac{|\alpha|^{2}(1+e(x))-2 \alpha(\operatorname{Re} \alpha) e(x)-i \bar{A} \bar{\alpha} e^{2 i \alpha x}}{|\alpha|^{2}(1-e(x))} .
$$

If $\operatorname{Im}(1+\Delta(x, x))=0$ and $\operatorname{Re}(1+\Delta(x, x)) \leq 0$ at some $x \geq 0$, then

$$
\left\{\begin{array}{l}
\operatorname{Re}\left(\bar{A} \bar{\alpha} e^{2 i \alpha x}\right)=-2(\operatorname{Im} \alpha)(\operatorname{Re} \alpha) e(x) \\
-\operatorname{Im}\left(\bar{A} \bar{\alpha} e^{2 i \alpha x}\right) \geq|\alpha|^{2}(1+e(x))-2(\operatorname{Re} \alpha)^{2} e(x)>0 .
\end{array}\right.
$$

However, this is impossible since, at this $x$,

$$
\begin{aligned}
|A|^{2}|\alpha|^{2} e^{-4(\operatorname{Im} \alpha) x} & =\left(\operatorname{Re}\left(\bar{A} \bar{\alpha} e^{2 i \alpha x}\right)\right)^{2}+\left(\operatorname{Im}\left(\bar{A} \bar{\alpha} e^{2 i \alpha x}\right)\right)^{2} \\
& \geq 4(\operatorname{Im} \alpha)^{2}(\operatorname{Re} \alpha)^{2} e(x)^{2}+\left(|\alpha|^{2}(1+e(x))-2(\operatorname{Re} \alpha)^{2} e(x)\right)^{2} \\
& =|\alpha|^{4}(1-e(x))^{2}+4|\alpha|^{2}(\operatorname{Im} \alpha)^{2} e(x) \\
& =|\alpha|^{4}(1-e(x))^{2}+|A|^{2}|\alpha|^{2} e^{-4(\operatorname{Im} \alpha) x} \\
& \geq|A|^{2}|\alpha|^{2} e^{-4(\operatorname{Im} \alpha) x} .
\end{aligned}
$$

Therefore the curve $z(x)=1+\Delta(x, x), x \geq 0$, in $C$ can not intersect the half line $z \leq 0$, and so,

$$
\left[\arg (1+\Delta(x, x)]_{0}^{\infty}\right.
$$

takes a value in $(-\pi, \pi)$. This implies that $\Delta(x, y)$ satisfies condition (S4) if and only if $\operatorname{Im}(1+\Delta(0,0))=0$, because, by (5.3), the constant $C$ equals 1 for $S(k)$ in (5.1). Since 


$$
\begin{aligned}
\operatorname{Im}(1+\Delta(0,0))=0 & \Leftrightarrow 2(\operatorname{Im} \alpha)(\operatorname{Re} \alpha) e(0)+\operatorname{Re}(\bar{A} \bar{\alpha})=0 \\
& \Leftrightarrow(\operatorname{Re} \alpha)\left|\frac{\bar{\beta}-\bar{\alpha}}{\bar{\alpha}-\beta}\right|^{2}+\operatorname{Re}\left(\bar{\alpha} \frac{\beta-\alpha}{\alpha-\bar{\beta}}\right)=0 \\
& \Leftrightarrow \operatorname{Re}\left(\bar{\alpha}+\bar{\alpha}\left(\frac{\bar{\alpha}-\beta}{\bar{\beta}-\bar{\alpha}}\right)\right)=0 \\
& \Leftrightarrow \overline{\left(\frac{\beta}{\alpha}\right)}=\frac{\beta}{\alpha},
\end{aligned}
$$

the condition $\operatorname{Im}(1+\Delta(0,0))=0$ is rewritten as $\beta=c \alpha(c>0)$.

Let $\beta=c \alpha$ with $c>0$. Without loss of generality we assume that $\operatorname{Im} \alpha=1 / 2$. Then, by setting $\kappa=\operatorname{Re} 2 \alpha$, we have

$$
\begin{gathered}
\alpha=\frac{1}{2}(\kappa+i), \quad|\alpha|^{2}=\frac{1+\kappa^{2}}{4}, \quad A=\frac{(c-1)\left\{\kappa^{2}(1-c)+(1+c)+2 \kappa c i\right\}}{\kappa^{2}(1-c)^{2}+(1+c)^{2}}, \\
|A|=\frac{\left(1+\kappa^{2}\right)|1-c|}{\sqrt{\kappa^{2}(1-c)^{2}+(1+c)^{2}}}, \quad e(x)=\frac{\left(1+\kappa^{2}\right)(1-c)^{2}}{\kappa^{2}(1-c)^{2}+(1+c)^{2}} e^{-2 x} .
\end{gathered}
$$

The fundamental function $\Delta(x, t)$ is determined from (5.9). Hence a pair $(Q, U)$ of potentials for which the scattering data is $S(k)$ in $(5.1)$ can be obtained by (1.5), (1.6).

\section{References}

[1] Agranovich, Z. S. and Marchenko, V. A., The Inverse Problem of Scattering Theory, Gordon and Breach, New York, 1963.

[2] Aktosun, T., Klaus, M. and van der Mee, C., Wave scattering in one dimension with absorption, J. Math. Phys., 39 (1998), 1957-1992.

[3] Aktosun, T., Klaus, M. and van der Mee, C., Inverse scattering in one-dimensional nonconservative media, Integral Equations Operator Theory, 30 (1998), 279-316.

[4] Chadan, K. and Sabatier, P. C., Inverse Problems in Quantum Scattering Theory, 2nd ed., Springer-Verlag, New York, 1989.

[5] Cornille, H., Existence and uniqueness of crossing symmetric $N / D$-type equations corresponding to the Klein-Gordon equation. J. Math. Phys., 11 (1970), 79-98.

[6] Jaulent, M., On an inverse scattering problem with an energy-dependent potential, Ann. Inst. Henri Poincaré, Sect A, 17 (1972), 363-378.

[7] Jaulent, M., Sur le problème inverse de la diffusion pou l'équation de Shcrödinger radiale avec un potentiel dépendent de l'énrgie, C. R. Acad. Sc. Paris, 280 (1975), 1467-1470.

[ 8 ] Jaulent, M., Inverse scattering problems in absorbing media, J. Math. Phys., 17 (1976), 1351-1360.

[9] Jaulent, M. and Jean, C., The inverse s-wave scattering problem for a class of potentials depending on energy, Comm. Math. Phys., 28 (1972), 177-220. 
[10] Jaulent, M. and Jean, C., The inverse problem for the one-dimensional Schrödinger equation with an energy-dependent potential, I, II, Ann. Inst. Henri Poincaré, Sect A, 25 (1976), 105$118,119-137$.

[11] Kamimura, Y., An inversion formula in energy dependent scattering, J. Integral Equations Appl., 19 (2007), 473-512.

[12] Kamimura, Y., An inverse problem in advection-diffusion, J. Phys., Conf. Ser., 54 (2007), doi 012012.

[13] Kaup, D. J., A higher-order water-wave equation and the method for solving it, Progr. Theoret. Phys., 54 (1975), 396-408.

[14] Marchenko, V. A., Sturm-Liouville Operators and Applications, Operator Theory: Advances and Applications, 22, Birkhäuser-Verlag, Basel, 1986.

[15] Paley, R. E. A. C. and Wiener, N, Fourier Transforms in the Complex Domain, Colloquium Publication, 19, Amer. Math. Soc., 1934.

[16] Sattinger, D. H. and Szmigielski, J., Energy dependent scattering theory, Differential Integral Equations, 8 (1995), 945-959.

[17] Sattinger, D. H. and Szmigielski, J., A Riemann-Hilbert problem for an energy dependent Schrödinger operator, Inverse Problems, 12 (1996), 1003-1025.

[18] van der Mee, C. and Pivovarchik, V., Inverse scattering for a Schrödinger equation with energy dependent potential, J. Math. Phys., 42 (2001), 158-181.

\author{
nuna adreso: \\ Department of Ocean Sciences \\ Tokyo University of Marine Science and \\ Technology \\ Minato-ku, Tokyo 108-8477 \\ Japan \\ E-mail: kamimura@kaiyodai.ac.jp
}

(Ricevita la 21-an de novembro, 2006) 